\title{
FORESTACIÓN DE ISLETAS DE DRAGADO UTILIZANDO DOS ESPECIES DE MANGLES, EN UNA LAGUNA COSTERA DEL Golfo de California, MÉXico
}

\author{
Daniel Benitez-Pardo', Francisco Javier Flores-Verdugo², Margarita Casas-Valdez ${ }^{3}$, \\ Gustavo Hernández-Carmona ${ }^{3}$, Juan Ignacio Valdez-Hernández ${ }^{4}$ \\ y VÍCTOR GÓMEZ-MUÑOZ \\ ${ }^{1}$ Facultad de Ciencias del Mar de la Universidad Autónoma de Sinaloa, Mazatlán, Sinaloa, México \\ ${ }^{2}$ Unidad Mazatlán, Instituto de Ciencias del Mar y Limnología de la Universidad Nacional Autónoma de México, \\ Mazatlán, Sinaloa, México \\ ${ }^{3}$ Centro Interdisciplinario de Ciencias Marinas del Instituto Politécnico Nacional, La Paz, Baja California Sur, México \\ ${ }^{4}$ Colegio de Postgraduados, Campus Montecillo, Texcoco, Estado de México, México \\ ${ }^{1}$ Autor para la correspondencia: dbpardo6@hotmail.com
}

\begin{abstract}
Resumen: El propósito del presente estudio fue generar información en torno a la posibilidad de estabilizar isletas de dragado mediante la plantación de halófitas, particularmente con la creación de nuevas áreas de manglar. Para esto, fue construido un vivero para producir plantas de Avicennia germinans y Rhizophora mangle, a la vez que se obtuvieron individuos del medio natural para ambas especies. Para su plantación se consideró el intervalo de mareas, áreas de baja energía, salinidad, zonación de cada especie y un diseño experimental de bloques completos al azar. Los resultados indicaron una mayor supervivencia y crecimiento de las plantas de vivero que las extraídas del medio natural. La supervivencia de plantas de A. germinans ( $78 \%$ con contenedor, $72 \%$ sin contenedor, $34 \%$ medio natural) fue mayor que la de $R$. mangle (43\% con contenedor, $34 \%$ sin contenedor, $21 \%$ medio natural). De igual manera, el crecimiento en altura de las plantas de $A$. germinans, tanto de las producidas en vivero $\left(3.2 \mathrm{~cm} \mathrm{mes}^{-1}\right)$, como las extraídas del medio natural $\left(1.5 \mathrm{~cm} \mathrm{mes}^{-1}\right)$, fue mayor que las de $R$. mangle (producidas en vivero: $1.4 \mathrm{~cm} \mathrm{mes}^{-1}$; extraídas del medio natural: $0.8 \mathrm{~cm} \mathrm{mes}^{-1}$ ). No se presentó colonización natural durante el periodo de estudio, por lo que son recomendables las plantas de vivero para iniciar la forestación de isletas artificiales, considerando las mareas, la zonación natural local, áreas de baja energía y sobre todo, el hidroperiodo.
\end{abstract}

Palabras claves: Avicennia germinans, forestación, hidroperiodo, isletas artificiales, Rhizophora mangle.

Abstract: The purpose of this study is on digging islands created with dredge material. A nursery station was generated in order to produce propagules of Avicennia germinans and Rhizophora mangle. Tide amplitude, low energy zones, salinity, and mangrove zonation were considered for propagules recruitment within random experimental quadrants on the islands. Survival rates of $A$. germinans were $78 \%$ and $72 \%$ for plants with container and without container, respectively. Rhizophora mangle showed a $43 \%$ and $34 \%$ for the same parameters. Plants extracted from their natural environment presented a survival rate of $34 \%$ for A. germinans, and $21 \%$ for $R$. mangle. Avicennia germinans growth from nursery station presented $3.2 \mathrm{~cm} / \mathrm{month}$, while plants from their natural environment $1.5 \mathrm{~cm} /$ month. Rhizophora mangle showed $1.4 \mathrm{~cm} / \mathrm{month}$ in comparison with natural environment with 0.8 $\mathrm{cm} / \mathrm{month}$. Results showed a higher growth and survival rate of plants from nursery station compared to plants from the natural environment. There was no natural recruitment during the study; therefore, nursery station mangrove plants should be used in artificial islands considering tide amplitude, natural zonation, low energy zones, and the hydroperiod.

Key words: Avicennia germinans, dredge soil islands, forestation, hydroperiod, Rhizophora mangle.

$\mathbf{E}^{\prime}$ manglar es un ecosistema costero que presenta importancia ecológica, económica, social y cultural; ofrece protección, alimentación y reproducción para diversos organismos acuáticos, muchos de ellos con interés comercial
(Harrison et al., 1994; Tovilla, 1994; Moreno et al., 2002; Creary, 2003; Kieckbusch, 2004). Se estima que por cada hectárea de manglar destruida se pierden anualmente alrededor de $800 \mathrm{~kg}$, entre camarones y peces, de importancia 
comercial (Turner, 1977; Acosta y Butler, 1997). Las actividades humanas destruyeron en 25 años (1980 a 2005), alrededor de 4.6 millones de hectáreas de manglares en el mundo, el 23\% de la cobertura total original (FAO, 2007a, b). Particularmente, de 1970 al 2004, la acuacultura ha desforestado aproximadamente 544,000 ha de manglar en países como Bangladesh, Brasil, China, Ecuador, India, Tailandia y Vietnam (Hamilton, 2013).

No obstante que existe información concerniente a las medidas y técnicas apropiadas para la recuperación de manglares afectados en Florida, E.U.A. (Lewis, 1982), así como en Colombia, Guatemala, Nicaragua y Panamá (Elster, 2000), muchos de los proyectos se han centrado en especies del género Rhizophora u otros de la familia Rhizophoraceae (Komiyama et al., 1996), y se han considerado pocas veces especies de mangles de otras familias (p.ej. Acanthaceae) y géneros (p.ej. Avicennia). En México, la recuperación de manglares ha seguido tres tendencias: reforestación, restauración hidrológica y combinación de ambas (Flores-Verdugo et al., 2006). La mayoría de la información publicada se refiere a la primera tendencia, con ejemplos en zonas cálido húmedas y subhúmedas de Campeche, Chiapas, Colima y Yucatán (ZaldivarJiménez et al., 2000; Tovilla y Orihuela, 2002; Flores-Verdugo et al., 2006; Agraz-Hernández, 2007).

Sin embargo, en zonas semiáridas de México (p.ej. costas del Golfo de California). Los trabajos de recuperación de manglares son escasos (Toledo et al., 2001). En estas zonas no existe disponibilidad de propágulos durante todo el año y su siembra directa está limitada por efecto de las mareas (Paré y Fraga, 1994; Agraz-Hernández, 1999; Reyes y Tovi1la, 2002). Además, el azolvamiento de las lagunas costeras, calculado en promedio en $1 \mathrm{~cm}$ año-1 (Ruiz-Fernández et al., 2003), representa un problema importante que afecta directamente al ecosistema del manglar. Bajo estas condiciones, las isletas creadas al depositar el producto de los dragados en el interior de las lagunas (SEMARNAP, 2000) constituyen zonas viables de plantación de mangles, los cuales pueden obtenerse de viveros forestales, que han mostrado una mayor supervivencia en comparación con la siembra directa de propágulos (Elster, 2000).

Por tal razón, el objetivo del presente estudio fue registrar las condiciones ambientales (agua, sustrato) y medir las características de las plantas (supervivencia, altura, diámetro) de las especies Avicennia germinans (L.) L. (Acanthaceae) y Rhizophora mangle L. (Rhizophoraceae), establecidas en isletas de dragado en una laguna costera del Golfo de California. Se espera, particularmente, contribuir al conocimiento de la creación de nuevas áreas de manglar al evaluar su desempeño en campo; para lo cual, algunas preguntas relevantes son: ¿el crecimiento de las plantas es igual en los dos niveles topográficos?, ¿las plantas obtenidas de vivero tienen mayor supervivencia que aquellas del medio natural?

\section{Materiales y Métodos}

Sitio de estudio. Este trabajo se realizó en una isleta producto de dragados, ubicada a $25^{\circ} 29^{\prime} 34.23^{\prime \prime}$ N y $108^{\circ} 47^{\prime} 24.36$ " $\mathrm{O}$, en la parte interna de la bahía de Navachiste, localizada en el noroeste de México, entre los $25^{\circ} 10^{\prime}$ y $25^{\circ} 22^{\prime} \mathrm{N}$ y $109^{\circ} 00^{\prime}$ y $108^{\circ} 40^{\prime} \mathrm{O}$ (Figura 1). Presenta un clima que va desde el tipo semiseco muy cálido (BS h') al muy seco y muy cálido (BW h'). La temperatura promedio se mantiene por encima de los $18{ }^{\circ} \mathrm{C}$, y los meses más cálidos son julio y agosto, los que registran temperaturas máximas de $38{ }^{\circ} \mathrm{C}$ y $45^{\circ} \mathrm{C}$, respectivamente (García, 1980). La precipitación media anual oscila entre los 365 y $450 \mathrm{~mm}$; sin embargo, la evaporación promedio anual es cercana a los 2,000 mm (SEMARNAP, 2000).

Construcción de isletas de dragado. La construcción de las isletas estuvo a cargo de la Comisión Nacional de Acuacultura y Pesca (CONAPESCA, 2001). Las obras iniciaron en 2003 y, para su ubicación, se consideraron aquellos bajos de mareas sin importancia para la pesca de moluscos y/o sobrexplotados, así como una distancia mínima de $50 \mathrm{~m}$ entre la zona de manglar más cercana y las islas naturales. El experimento se inició nueve meses después de haber construido las isletas, para tener un menor efecto de subsidencia del sustrato.

Sitios experimentales. Los sitios seleccionados para el experimento se consideraron de acuerdo con las mareas y la microtopografía local, la salinidad intersticial, las zonas de baja energía del viento y el oleaje. Los sitios específicos de trasplante de cada especie se determinaron de acuerdo con la zonación local (niveles topográficos); es decir, donde se encuentran establecidas cada una de las especies de manera natural: Rhizophora mangle en la zona más inundada y Avicennia germinans en la menos inundada; de esta forma se marcan dos niveles topográficos. A partir de la zonación o franja natural de manglar, los niveles se trasladaron hasta la isleta con el apoyo de un topógrafo, de esta manera se determinó las franja intermareal donde se llevó a cabo la forestación (salinidad intersticial de 35 a $70 \mathrm{~g} \mathrm{~kg}^{-1}$ ).

Plantas de vivero para la forestación. Se construyó un vivero para producir más de 25,000 plantas de las especies Avicennia germinans y Rhizophora mangle, utilizando bolsas (contenedores) de polietileno de $18 \mathrm{~cm}$ de diámetro por $28 \mathrm{~cm}$ de largo. Las bolsas fueron llenadas con una mezcla de $80 \%$ de sustrato de rivera de río y $20 \%$ de arena; para reducir la intensidad de la luz solar se utilizó un techo de malla-sombra al $60 \%$ de sombra. Cabe señalar que los mangles, antes de ser trasplantados a la zona experimental, se sometieron a un proceso de aclimatación, el cual consistió en quitar un mes antes la malla-sombra que los cubría en el vivero y regarlos durante diez días con cada una de las si- 
guientes concentraciones de salinidad en el agua: $10 \mathrm{~g} \mathrm{~kg}^{-1}$, $20 \mathrm{~g} \mathrm{~kg}^{-1}$ y $30 \mathrm{~g} \mathrm{~kg}^{-1}$.

Diseño experimental. Los tratamientos se conformaron de acuerdo con la especie (Avicennia germinans, Rhizophora mangle), y forma de obtención de las plantas (producidas en vivero con y sin contenedor, extraídas del medio natural), quedando de la siguiente manera: 50 plantas de A. germinans con contenedor $(\mathrm{Agn}+\mathrm{C})$ y 50 sin contenedor (Agn-C), 50 plantas de $R$. mangle con contenedor $(\mathrm{Rmn}+\mathrm{C})$ y $50 \mathrm{sin}$ contenedor (Rmn-C), de diez meses de edad para ambas especies; 50 plantas de A. germinans extraídas del medio natural $(\mathrm{AgNa})$ y 50 de $R$. mangle $(\mathrm{RmNa})$, también extraídas del medio natural, ambas con aproximadamente diez meses de edad. Los tratamientos fueron plantados en cada uno de los dos niveles topográficos de acuerdo con la distribución natural del manglar local (zonación). El Nivel 1 correspondió a la zona o franja menos inundada (aproximadamente $40 \mathrm{~cm}$ ) y el Nivel 2 a la más inundada (aproximadamente 60 $\mathrm{cm})$. La distribución de las hileras de plantas se hizo al azar dentro de cada nivel topográfico, utilizando el Diseño de Bloques Aleatorizados (DBA) con un arreglo factorial $2 \times 2$ $\times 3$ (dos especies, dos niveles topográficos y tres formas de obtención de las plantas), lo que conforma 12 tratamientos, con un tamaño de muestra de 50 plantas.

La plantación se llevó a cabo en agosto de 2004. Los mangles fueron plantados con una separación de $1.5 \mathrm{~m} \times 1.5 \mathrm{~m}$, por lo que se tuvo una densidad aproximada de 4,500 plantas por hectárea. Las plantas fueron depositadas en pozos de $20 \mathrm{~cm}$ de diámetro por, aproximadamente, $35 \mathrm{~cm}$ de profundidad. Al momento de la plantación, se le hizo un corte lon- gitudinal a la bolsa y al fondo. Se consideró la altura inicial de los individuos y bimensualmente, por un año, se tomaron datos de supervivencia, altura de la planta y diámetro del tallo a la altura del cuello de la planta.

Parámetros ambientales. Al agua superficial e intersticial se les determinó mensualmente la temperatura, salinidad $\left(\mathrm{g} \mathrm{kg}^{-1}\right), \mathrm{pH}$ y la conductividad eléctrica (CE) al inicio y al final del estudio. Para la toma de muestras de agua intersticial de ambos niveles topográficos, se cavaron hoyos cada 5 $\mathrm{m}$ a la profundidad suficiente para encontrar agua, esto fue a lo largo de transectos que se marcaron en el lote experimental considerando la dirección del gradiente topográfico. La temperatura se tomó con un termómetro de mercurio (Brannan Cumbria, Reino Unido). La salinidad se determinó mediante un refractómetro portátil (80-124, Handhel, Orem, Utah, EEUU). El pH y la CE se midieron con un potenciómetro portátil digital (Hanna HI 9813, Rumania). La granulometría del sustrato se determinó usando la técnica del hidrómetro de Boyoucos (Jackson, 1964).

Parámetros biológicos. La supervivencia de las plantas fue determinada mensualmente para conocer cuántas fenecían en cada tratamiento de cada especie durante el estudio. La altura de Avicennia germinans se determinó con la ayuda de un flexómetro de madera, graduado en mm; para ello se consideró desde el cuello de la planta hasta la inserción del último par de hojas verdaderas de la rama principal. Para Rhizophora mangle esta medida se hizo a partir de la parte apical del hipocótilo. El diámetro del tallo de A. germinans se obtuvo de medir el cuello de la planta un centímetro arri-

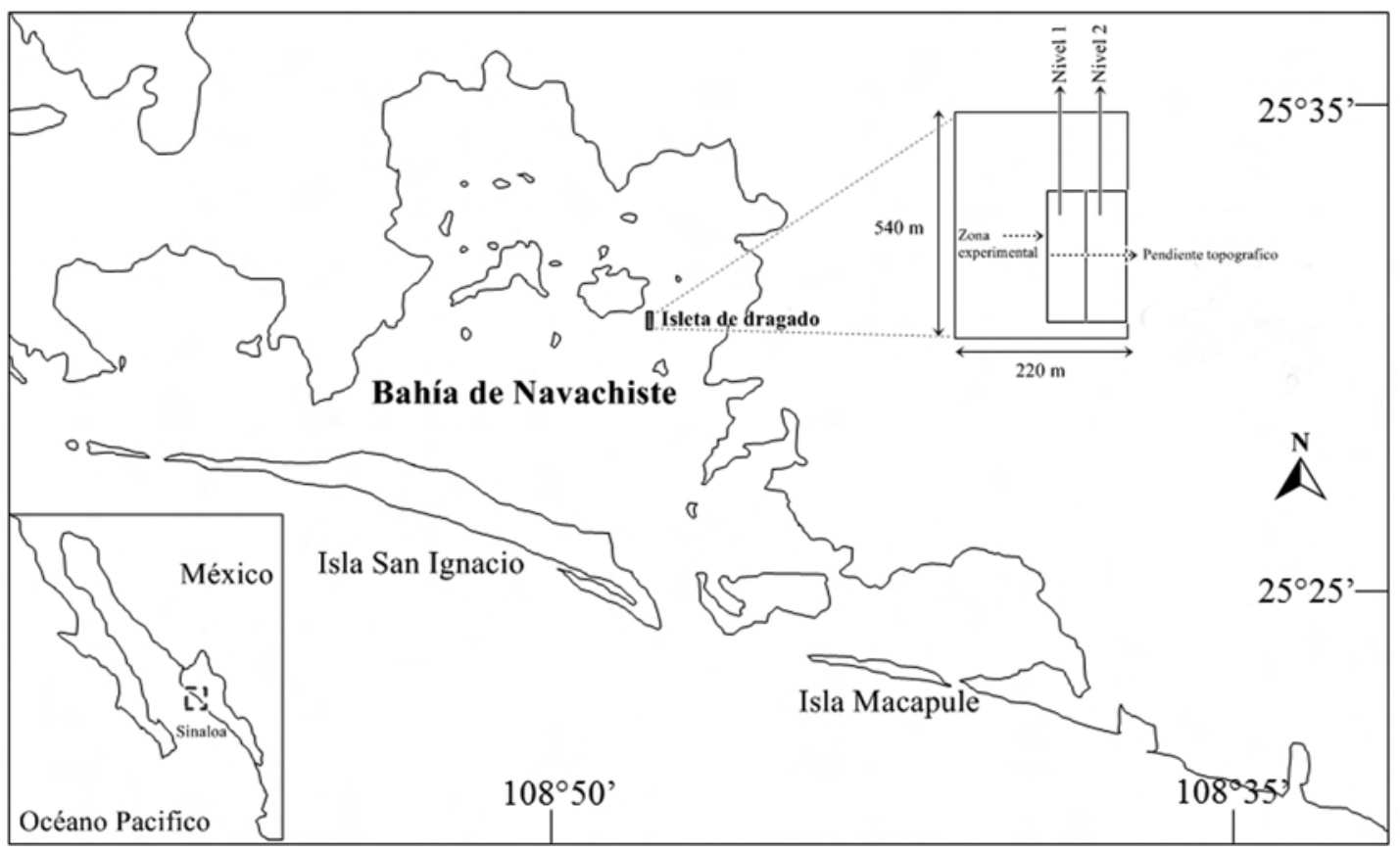

Figura 1. Sitio de estudio. Bahía de Navachiste, Sinaloa, noroeste de México. 
Daniel Benitez-Pardo et al.

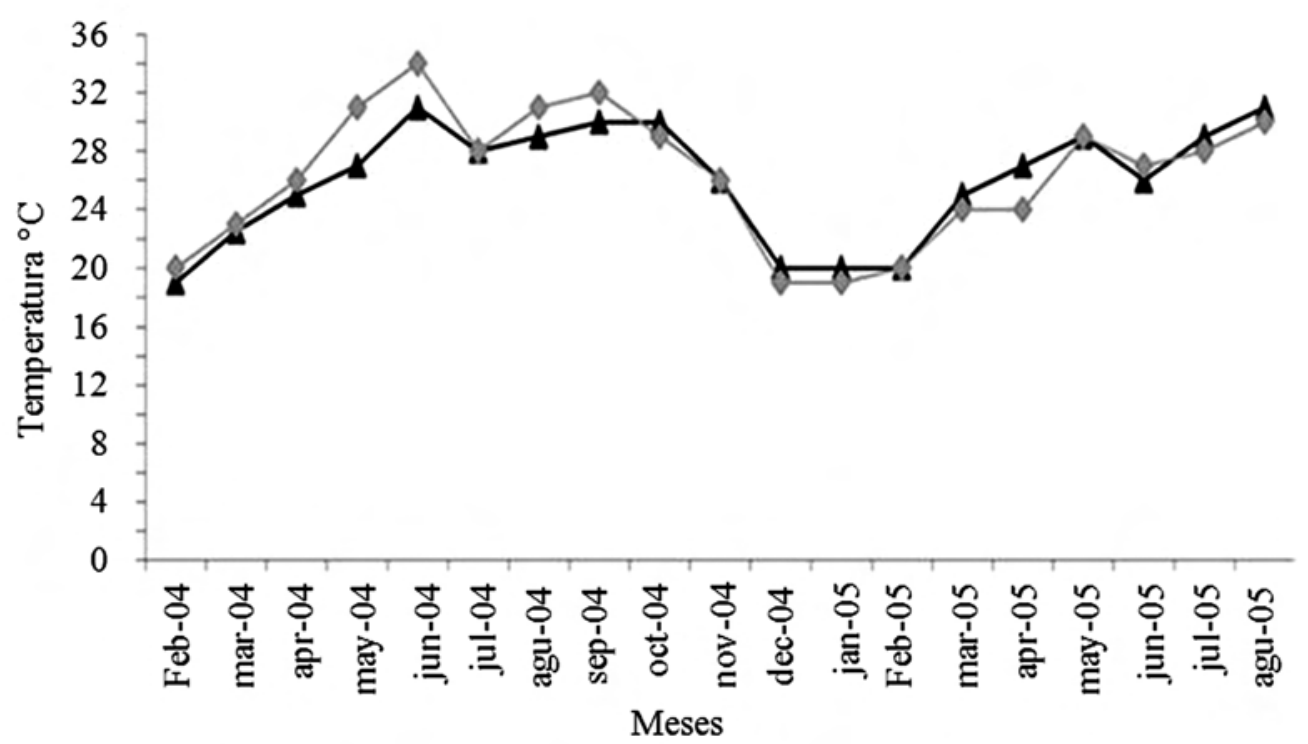

Figura 2. Temperatura del agua intersticial $(\boldsymbol{\Delta})$ y superficial $(\diamond)$ en la isleta de dragado en la Bahía de Navachiste, Sinaloa, noroeste de México.

ba de la superficie del suelo. Para $R$. mangle, se tomó como cuello de la planta, donde nace la rama principal en la parte apical del hipocótilo. Las medidas se tomaron con un vernier de plástico (W., Alemania).

Tamaño de raíces: Antes del establecimiento del experimento, se midió longitud y diámetro de la raíz principal a 200 plantas de Avicennia germinans y Rhizophora mangle de vivero, y a 200 plantas extraídas del medio natural. La longitud se midió con un flexómetro graduado en milímetros y el diámetro con vernier de plástico (W., Alemania).

Análisis estadístico. Para las variables "altura y diámetro de planta" se aplicó regresión múltiple a la variable "salinidad", respecto a los factores niveles topográficos y forma de obtención de las plantas, con su correspondiente análisis de Covarianza (ANCOVA). Para el caso de la variable "supervivencia” (datos no paramétricos), se les aplicó la prueba estadística Kruskal-Wallis (Mendenhall y Sincich, 1997). A las medias de los tratamientos que resultaron con diferencias significativas se les aplicó la prueba de comparaciones múltiples de Tukey al 5\% de probabilidad del error.

\section{Resultados}

Parámetros ambientales. La textura del suelo de la isleta de dragado presentó, al inicio del estudio, $62 \%$ de arena, $19 \%$ de arcilla y $19 \%$ de limo; mientras que al final del mismo, $57 \%$ de arena, $21 \%$ de arcilla y $22 \%$ de limo. La temperatura del agua superficial e intersticial mostró el patrón anual: las temperaturas más altas se observaron en mayo, junio y julio, las más bajas en diciembre, enero y febrero, para ambos casos (Figura 2).
La salinidad presentó variación en función al gradiente topográfico; por ejemplo, en el punto (pozo) de muestreo uno, los datos van desde, aproximadamente, $50 \mathrm{~g} \mathrm{~kg}^{-1}$ hasta $190 \mathrm{~g} \mathrm{~kg}^{-1} \mathrm{y}$, a medida que disminuye la pendiente topográfica, los resultados se asemejan a la salinidad del agua su-

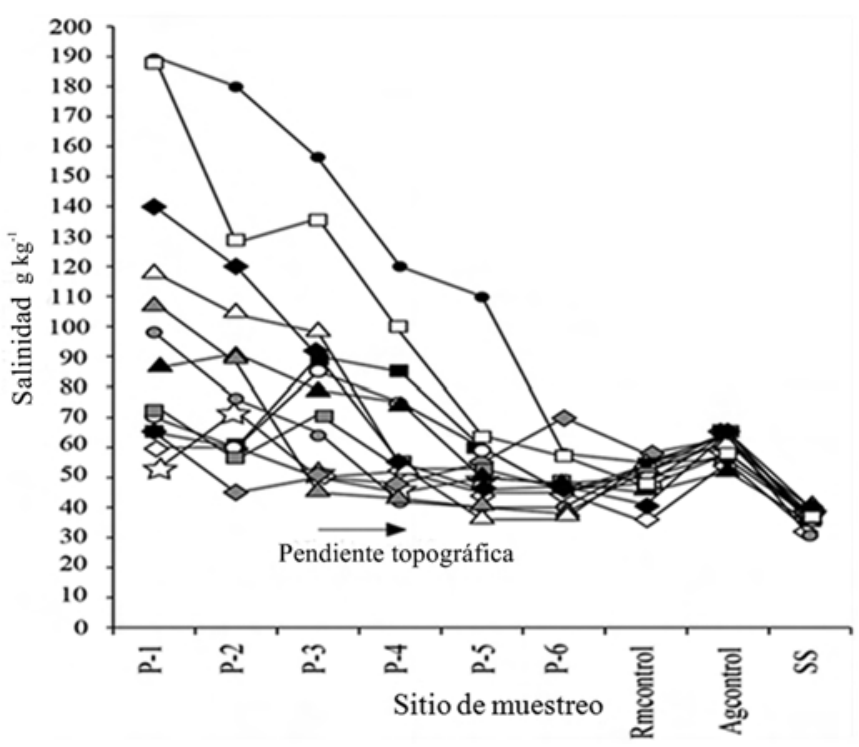

Figura 3. Salinidad intersticial del gradiente topográfico en el área experimental de la isleta de dragado en la Bahía de Navachiste, Sinaloa, noroeste de México. P1 = muestra sitio 1 (pozo), P2 = muestra sitio $2, \mathrm{P} 3=$ muestra sitio $3, \mathrm{P} 4=$ muestra sitio $4, \mathrm{P} 5=$ muestra sitio 5, P6 = muestra sitio 6, Rmcontrol $=$ Rhizophora mangle control, Agcontrol = Avicennia germinans control, $\mathrm{SS}=$ salinidad superficial. $\Delta$ Agosto 2004; Sep 2004; $\boldsymbol{\Delta}$ Octubre 2004; Noviembre 2004; $\diamond$ Dic 2004; the enero 2005; $\diamond$ Febrero 2005; $\square$ Marzo 2005; • abril 2005; $\Delta$ Mayo 2005; $\diamond$ Junio 2005; - Julio 2005; O Agosto 2005.

Botanical Sciences 93 (1): 165-174, 2015 
perficial, entre 33 y $45 \mathrm{~g} \mathrm{~kg}^{-1}$ durante el tiempo de muestreo (Figura 3). En contraste, en los puntos de control los valores fluctuaron de 55 a $65 \mathrm{~g} \mathrm{~kg}^{-1}$ para Avicennia germinans y de 48 a $58 \mathrm{~g} \mathrm{~kg}^{-1}$ para Rhizophora mangle (Figura 3).

El pH del sustrato del sitio experimental fue de 7.4 al inicio del trabajo (agosto 2004) y de 8.2 al final del mismo (agosto 2005). En cambio, el pH del agua intersticial fue mayor que el del sustrato, pero muy similar tanto al inicio (8.3) como al final (8.4) del estudio, al igual que en los puntos de control, tanto para Avicennia germinans (8.4) como para Rhizophora mangle (8.6). La CE del agua intersticial fue $56 \mathrm{dS} \mathrm{m}^{-1}$ al inicio del estudio y $63 \mathrm{dS} \mathrm{m}^{-1}$ al final del mismo, valores menores para A. germinans $\left(122 \mathrm{dS} \mathrm{m}^{-1}\right) \mathrm{y}$ $R$. mangle $\left(80.5 \mathrm{dS} \mathrm{m}^{-1}\right)$ fueron obtenidos en los puntos de control.

Parámetros biológicos. Después de un año, la supervivencia de plantas de Avicennia germinans obtenidas de vivero con contenedor $(\mathrm{Agn}+\mathrm{C})$ y sin contenedor $(\mathrm{Agn}-\mathrm{C})$, así como de plantas extraídas del medio natural ( $\mathrm{AgNa})$, fue más alta $(P<0.05)$ en comparación con las plantas de Rhizohpora mangle obtenidas de vivero con contenedor $(\mathrm{Rmn}+\mathrm{C})$ y sin contenedor (Rmn-C), así como de las extraídas del medio natural (RmNa; Figura 4). De la misma forma, la supervivencia de plantas de vivero, tanto de A. germinans (75\%) como de $R$. mangle $(38 \%)$, fue más alta $(P<0.05)$ en comparación con las plantas de A. germinans (32\%) y R. mangle (22\%) obtenidas del medio natural (Figura 4). Por el contrario, no hubo diferencia significativa entre plan-

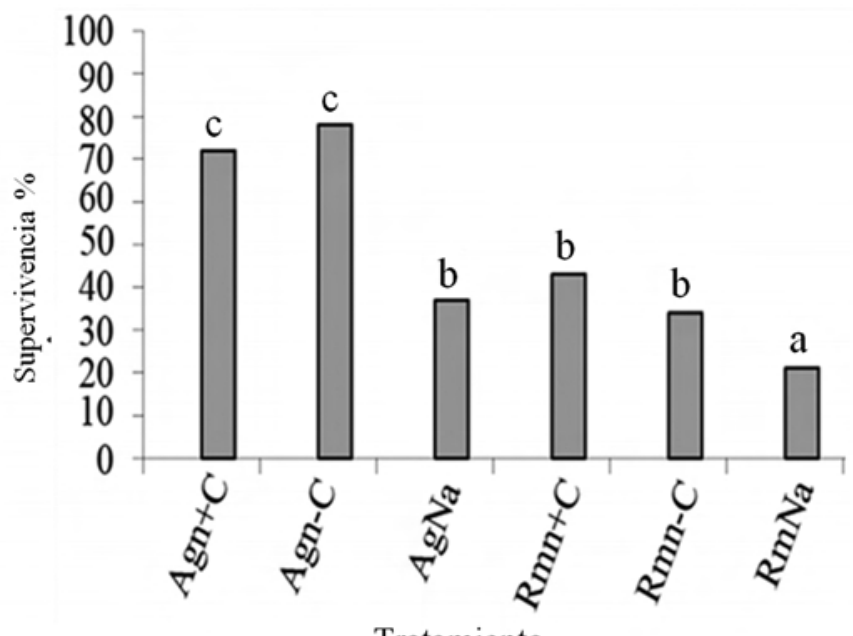

Tratamiento

Figura 4. Porcentaje de supervivencia de mangles después de dos años, en la isleta de dragado en la Bahía de Navachiste, Sinaloa, noroeste de México. Agn $+\mathrm{C}=$ Avicennia germinans de vivero con contenedor, Agn- $\mathrm{C}=A$. germinans de vivero sin contenedor, $\mathrm{AgNa}$ $=A$. germinans colectada en su medio natural, $\mathrm{Rmn}+\mathrm{C}=$ Rhizophora mangle de vivero con contenedor, $\mathrm{Rmn}-\mathrm{C}=R$. mangle de vivero sin contenedor y $\mathrm{RmNa}=R$. mangle colectada en su medio natural.

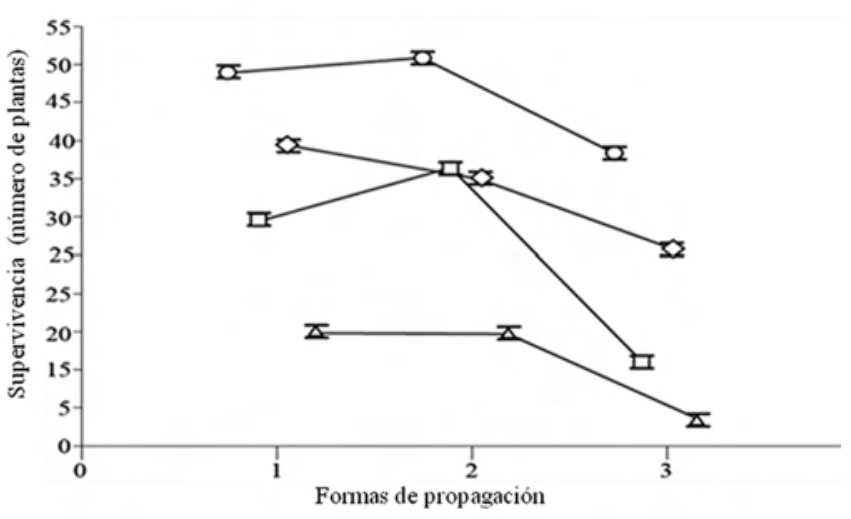

Figura 5. Supervivencia de Avicennia germinans y Rhizophora mangle en relación con los niveles topográficos y formas de propagación, en la isleta de dragado en la Bahía de Navachiste, Sinaloa noroeste de México. $\bar{\nabla}=$ Avicennia germinans con sus tres formas de propagación en el nivel topográfico $2, \overline{\mathrm{Q}}=A$. germinans con sus tres formas de propagación en el nivel topográfico $1, \square=$ Rhizophora mangle con sus tres formas de propagación en el nivel

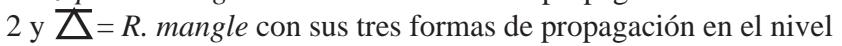
1. 1 = plantas de vivero con contenedor, $2=$ plantas de vivero sin contenedor, $3=$ plantas extraídas de su medio natural (testigo).

tas de vivero con y sin contenedor, entre ambas especies de mangles $(P>0.05)$.

De acuerdo con las pruebas de Kruskal-Wallis, hubo diferencias significativas $(P<0.05)$ entre la supervivencia de plantas establecidas en los diferentes niveles topográficos y entre las formas de obtención de las mismas, tanto para Avicennia germinans como para Rhizophora mangle (Figura 5). La supervivencia de $A$. germinans fue más alta $(P<$ 0.05 ) en el nivel topográfico 1 , que en el nivel topográfico 2 (46 \pm 5.75 vs. $26 \pm 16.6$ plantas). Respecto a las formas de obtención de las plantas, la supervivencia fue altamente significativa $(P<0.05)$ en ambos niveles topográficos, con el siguiente orden de los tratamientos: Agn-C ( $42 \pm 6$ plantas), Agn $+\mathrm{C}(32 \pm 9$ plantas $)$ y AgNa $(11 \pm 17$ plantas; Figura $5)$. En $R$. mangle, la supervivencia también fue mayor $(P$ $<0.05)$ en el nivel topográfico $1(38 \pm 7)$ que en el $2(26 \pm$ $10)$, existiendo diferencias $(P<0.05)$ con relación a las formas de obtención de las plantas entre niveles topográficos. La supervivencia en los tratamientos Rmn+C $(15 \pm 13)$ y Rmn-C $(15 \pm 15)$ fue diferente $(P<0.05)$ de la registrada en RmNa (4 \pm 17; Figura 5).

Para Avicennia germinans, la altura de las plantas no mostró diferencias significativas $(P>0.05)$ entre niveles topográficos, pero sí $(P<0.05)$ entre formas de obtención de las plantas (Figura 6). El crecimiento final en altura de $A$. germinans fue $26.5 \pm 2 \mathrm{~cm}$ en Agn-C y $24 \pm 2 \mathrm{~cm}$ en AgNa. Por el contrario, la altura de plantas en Rhizophora mangle fue diferente $(P<0.05)$ entre los niveles topográficos (Figura 6), registrándose el mayor valor en el nivel topográfico $2(14 \pm 4.4 \mathrm{~cm})$. El diámetro de las plantas no presentó diferencias significativas $(P>0.05)$ para $A$. germinans entre 


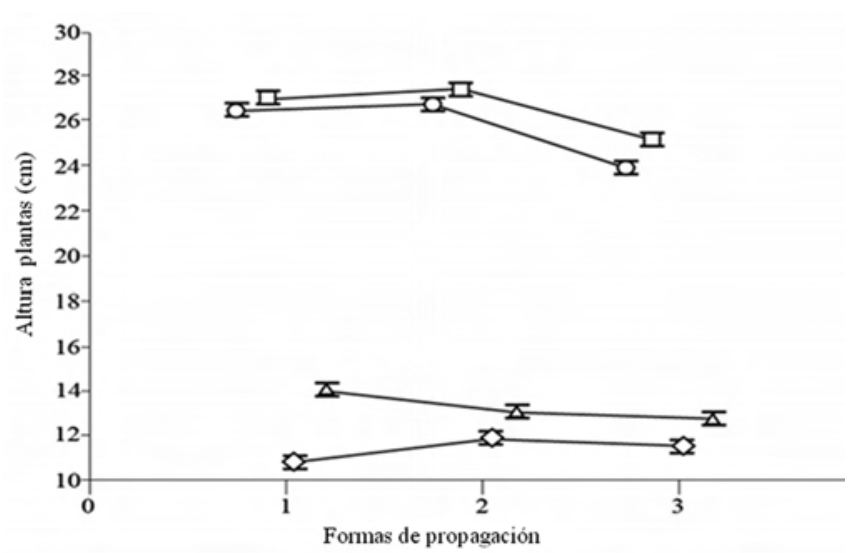

Figura 6. Altura de plantas de Avicennia germinans y Rhizophora mangle en relación con los niveles topográficos y formas de propagación, en la isleta de dragado en la Bahía de Navachiste, Sinaloa, noroeste de México. $\square=$ Avicennia germinans con sus tres formas de propagación en el nivel topográfico $2, \overline{\mathrm{V}}=$ A.germinans con sus tres formas de propagación en el nivel topográfico $1, \bar{\Delta}=$ Rhizophora mangle con sus tres formas de propagación en el nivel 2 y $\underline{\underline{ }}=$ R.mangle con sus tres formas de propagación en el nivel 1. $1=$ plantas de vivero con contenedor, $2=$ plantas de vivero $\sin$ contenedor, 3 = plantas extraídas de su medio natural (testigo).

niveles topográficos, pero si entre formas de obtención de las plantas $(P<0.05)$. En el nivel topográfico 1, el diámetro de planta de Agn+C $(6.3 \pm 3.2 \mathrm{~mm})$ fue mayor $(P<0.05)$ respecto a los tratamientos Agn-C $(5.7 \pm 2.2 \mathrm{~mm})$ y $\mathrm{AgNa}$ $(5.6 \pm 2.8 \mathrm{~mm})$. En el nivel 2, el diámetro de planta del tratamiento $\mathrm{AgNa}(5.6 \pm 2.7 \mathrm{~mm})$ fue bajo $(P<0.05)$ comparado con Agn+C (6.4 \pm 3.1$)$ y Agn-C (6.4 $\pm 3.2 \mathrm{~mm}$; Figura 7). En $R$. mangle, el diámetro de planta fue diferente $(P<0.05)$ entre niveles topográficos: $4.5 \pm 0.86 \mathrm{~mm}$ en el nivel $1 \mathrm{y}$ $5.6 \pm 2.9 \mathrm{~mm}$ en el nivel 2 (Figura 7). Los valores promedio en tamaño de raíces principales fueron, para $A$. germinans, $22 \mathrm{~cm}$ de longitud y $9.5 \mathrm{~cm}$ de diámetro en plantas de vivero, así como $10 \mathrm{~cm}$ de longitud y $7 \mathrm{~cm}$ de diámetro en plantas extraídas del medio natural; para $R$. mangle, $18 \mathrm{~cm}$ de longitud y $10.8 \mathrm{~cm}$ de diámetro en plantas de vivero, así como $9.2 \mathrm{~cm}$ de longitud y $6.5 \mathrm{~cm}$ de diámetro en plantas extraídas del medio natural.

\section{Discusión}

Parámetros ambientales. Los manglares crecen en sustratos cuya composición puede ser arenosa, lodosa, rocosa o calcárea (Furukawa y Wolanski, 1996). En la isleta de dragado, al iniciar el estudio predominaba un sustrato arenoso, pero con la dinámica de la isleta, dada por las condiciones ambientales locales y la sedimentación, el sustrato fue cambiando, en un periodo de dos años, su estructura granulométrica a menos arenosa con un ligero aumento en las arcillas y limos. Además, el gradiente topográfico de la isleta influyó en los procesos de óxido-reducción (Flores-Verdugo et al.,
2007), favoreciendo condiciones más propicias para el establecimientos de plantas (halófilas). También, la acumulación y sedimentación que determinan los procesos pedológicos cambiaron por efecto de las mareas, clima e hidrología. Según Furukawa y Wolanski (1996), los mangles se instalan donde existe una mayor acreción y sedimentación, con baja energía de oleaje y pendientes suaves, lo cual posiblemente contribuye a la colonización de plantas que ayudan a la estabilización y madurez del suelo en estos ambientes (Saenger, 2002).

La salinidad es un factor que determina la distribución vegetal en los humedales costeros (Cowardin et al., 1979). El hidroperiodo junto con la insolación tienen su efecto, de la misma manera, en la variación de la salinidad intersticial que determina la zonación de los manglares; la salinidad intersticial incrementa de acuerdo con el gradiente topográfico (Flores-Verdugo et al., 2007). Particularmente en la isleta de dragado, la salinidad intersticial varió entre 33 y $190 \mathrm{~g} \mathrm{~kg}^{-1}$. Los valores más altos se registraron en el nivel topográfico menos inundado (50 a $190 \mathrm{~g} \mathrm{~kg}^{-1}$ ) y los más bajos en el nivel topográfico más inundado (33 a $45 \mathrm{~g} \mathrm{~kg}^{-1}$ ). Estos valores en la salinidad intersticial, en la bahía de Navachiste, se deben a los gradientes topográficos, a la alta irradiación y evaporación (2,100 mm anual), pocas lluvias (510 $\mathrm{mm}$ anual) y a la ocurrencia de fuertes corrientes de mareas (Benitez-Pardo, 2007). La salinidad y luminosidad altas bajan la tasa de

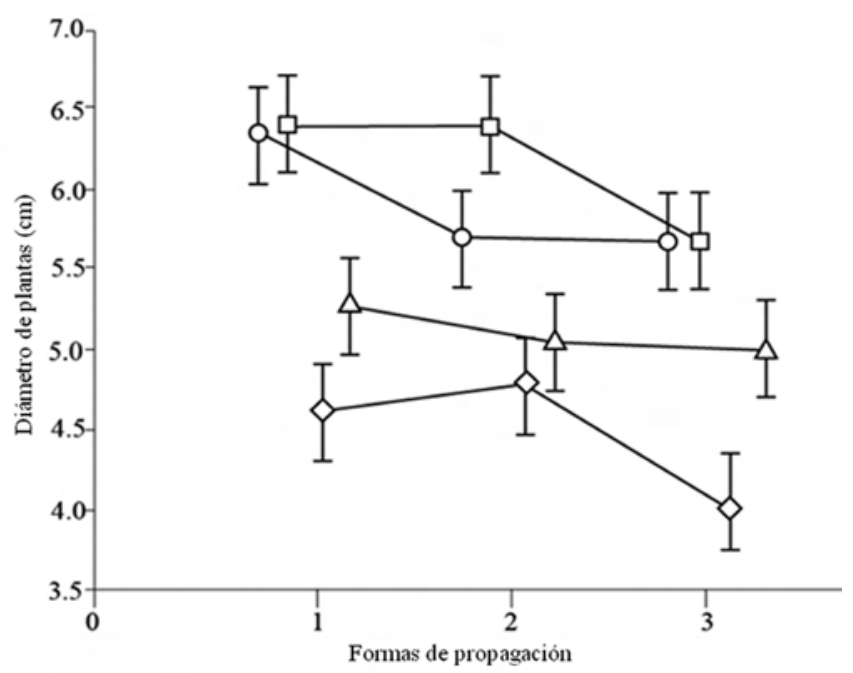

Figura 7. Diámetro de plantas de Avicennia germinans y Rhizophora mangle en relación con los niveles topográficos y formas de propagación, en la isleta de dragado en la Bahía de Navachiste, Sinaloa, noroeste de México. $\diamond=$ Rhizophora mangle con sus tres formas de propagación en el nivel topográfico $1, \triangle=R$. mangle con sus tres formas de propagación en el nivel topográfico 2,0 =Avicennia germinans con sus tres formas de propagación en el nivel topográfico 1 y $\square=$ A. germinans con sus tres formas de propagación en el nivel topográfico $2.1=$ plantas de vivero con contenedor, 2 = plantas de vivero sin contenedor, 3 = plantas extraídas de su medio natural (testigo). 
fotosíntesis y el metabolismo; además de aumentar la tasa de transpiración (Lichtenthaler, 1996). Sin embargo, debido a que los mangles son plantas halófilas facultativas, pueden sobrevivir en valores de salinidad que van de $1 \mathrm{~g} \mathrm{~kg}^{-1} \mathrm{a}<90 \mathrm{~g} \mathrm{~kg}^{-1}$ (Odum et al., 1982; Chapman, 1977). Contrariamente, la presencia de manglares en buen estado de desarrollo obedece a la existencia de una mezcla de agua dulce con marina $\left(15 \mathrm{~g} \mathrm{~kg}^{-1}\right)$, con aportes de nutrientes terrígenos por el agua dulce y la eliminación de competidores por el agua salada (Odum et al., 1982; Chapman, 1977).

Generalmente, los bosques monoespecíficos de Avicennia germinans se encuentran en $\mathrm{pH}$ de 8.4 y los de Rhizophora mangle en pH de 8.6 (Mckee et al., 1988; Chapman, 1977). En el sustrato del sitio experimental se encontró un $\mathrm{pH}$ de 7.4 al inicio del estudio y de 8.2 al final del mismo. Esto se puede explicar de la siguiente manera: al inicio, debido a la acidificación provocada por la exposición al aire del sustrato producto del dragado, que favorece la oxidación de elementos anaeróbicos del suelo (p.ej. de sulfuro a sulfato); y al final, debido a los carbonatos y bicarbonatos del agua de mar que incrementaron el $\mathrm{pH}$ hasta valores similares al agua de mar (8.1).

Otro factor determinante en el establecimiento de los mangles es el hidroperiodo, el cual depende principalmente del régimen local de mareas y de la microtopografía de los sitios (Flores-Verdugo et al., 2006, 2007). Los mangles prefieren aquellas planicies con gradientes topográficos suaves (microtopografía), debido a que, de manera natural, permiten que el agua salada o dulce penetre en los suelos del manglar y formen un gradiente de inundación (zonación) que resulta selectivo para cada especie (Monroy-Torres, 2005) de acuerdo con su tolerancia a la salinidad (Lugo y Cintron, 1975; Flores-Verdugo, 1989). El hidroperiodo combinado con la insolación produce que la salinidad intersticial aumente con el gradiente topográfico, lo cual se manifiesta en la altura de los manglares (Yang et al., 2013).

Con fines de establecimiento de plantaciones, es necesario conocer la elevación topográfica y el hidroperiodo para determinar en cuál zona las plantas tendrán más posibilidades de éxito (Mckee et al., 1988; Ellison et al., 2000). Por ejemplo, una inundación prolongada modifica la concentración de nutrientes en el sustrato, principalmente $\mathrm{N}$ y P (Ellison et al., 2000; Allen et al., 2003; Yang et al., 2013), lo cual afecta el desempeño de los individuos. Por tales razones, se tomó en cuenta el nivel topográfico y de inundación al momento de seleccionar la franja donde se estableció el experimento, junto con la zonación natural de Avicennia germinans y Rhizophora mangle. Sin embargo, el establecimiento de los mangles plantados ocurrió en una franja más angosta que la de los mangles locales, quizás debido a las condiciones microambientales y al dinamismo inicial que presentaron las isletas, así como al tamaño inicial de las plantas (aproximadamente $1 \mathrm{~m}$ ) y a la ausencia de raíces adventicias que hubiesen podido contribuir a la disipación de la energía del oleaje y de las corrientes mareales.

Otro de los factores determinantes para la distribución geográfica de los manglares es la temperatura, que oscila alrededor de los $20^{\circ} \mathrm{C}$ y no debe exceder un intervalo de $10^{\circ} \mathrm{C}$ (Chapman, 1977). En la bahía de Navachiste, la temperatura del agua, tanto superficial como intersticial, presentó variaciones estacionales similares a las reportadas por López-Aguiar (2006): para el verano fue de $33{ }^{\circ} \mathrm{C}$ y para invierno de $20^{\circ} \mathrm{C}$. Esto valores están dentro de los reportados para la distribución de manglares en el mundo (Tomlinson, 1986). En este mismo sentido, Clough (1992) encontró que los manglares son esencialmente tropicales, pero se adaptan a un intervalo amplio de temperatura; aunque algunas veces se distribuyen en los subtrópicos como sería el caso de la bahía de Navachiste. Este mismo autor señala que se han encontrado manglares en Australia desarrollándose en temperaturas de $15^{\circ} \mathrm{C}$ en promedio y $4{ }^{\circ} \mathrm{C}$ como mínimo en épocas de frío, también en ambientes entre $30^{\circ}$ y $40^{\circ} \mathrm{C}$ en épocas de baja precipitación.

Parámetros biológicos. La supervivencia y crecimiento de Avicennia germinans fueron mayores que los de Rhizophora mangle en la franja menos inundada (Nivel 1) y con mayor salinidad, lo cual coincide con reportes de supervivencia de A. germinans (97\%) para zonas con baja inundación en Costa Rica (Delgado et al., 2001). Otros resultados en la supervivencia de $A$. germinans semejantes a los del presente estudio, fueron obtenidos en la laguna de Balandra, Baja California Sur (74\%; Toledo et al., 2001), considerada, al igual que la bahía de Navachiste, una zona semiárida con altas concentraciones de salinidad. Esta tolerancia a la salinidad (Medina et al., 1995) puede deberse a que A. germinans es una especie excretora de sales (López-Portillo et al., 2005) que también tolera una mayor presión osmótica (Hellebust, 1976; Tomlinson, 1986; Salisbury y Ross, 1994) y conductividad eléctrica del agua intersticial (8 a $\left.14 \mathrm{dS} \mathrm{m}^{-1}\right)$,

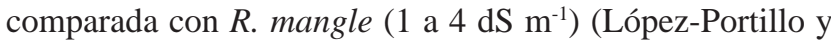
Ezcurra, 1989). Este resultado coincide con otros estudios de supervivencia y crecimiento de mangles en relación con la salinidad intersticial (Yan y Guizhu, 2007).

En la región de la bahía de Navachiste, la cobertura por Avicennia germinans representa entre el 65 y $70 \%$ de la superficie de manglar (Carrera y De la Fuente, 2003; Monzalvo-Santos, 2006). En este contexto, y considerando los resultados obtenidos, $A$. germinans es más viable que $R h i$ zophora mangle para la estabilización de isletas artificiales, lo cual puede deberse a la arquitectura de sus raíces (fijación al sustrato y geotropismo negativo), el desarrollo temprano de sus raíces adventicias (4 a 5 meses) y la mayor tolerancia a la salinidad (López-Portillo et al., 2005). Además, la alta protrusión o saliente radical de A. germinans (> 70\% en cinco días y $>80 \%$ en 12 días), le permite un mayor establecimiento de sus propágulos (Delgado et al., 2001). 
Las plantas de Avicennia germinans y Rhizophora mangle obtenidas de vivero, con y sin contenedor, presentaron mayor supervivencia que las extraídas del medio natural. Es posible que, tanto el tamaño de raíces al momento de la plantación, como el tamaño inicial de las plantas de vivero, fueran diferentes con respecto al de las extraídas del medio natural, a pesar de contar con la misma edad. Una supervivencia alta (> 80\%) se logra cuando las plantas tienen de 5 a $6 \mathrm{~mm}$ de diámetro (Sáenz et al., 2010). Las plantas extraídas del medio natural pudieron afectarse por una exposición directa a la luz solar, debido a que generalmente están protegidas por el dosel del manglar (Tomlinson, 1986), el cual permite un mayor establecimiento de propágulos al disminuir la desecación del sustrato (Tovilla y Orihuela, 2002; Bosire et al., 2003).

En la bahía de Navachiste, con salinidades de 36 y $50 \mathrm{~g} \mathrm{~kg}^{-1}$ en la época de lluvias y sequía, respectivamente, Avicennia germinans presentó mayor crecimiento que Rhizophora mangle, pero dicho crecimiento fue menor que el obtenido para lugares más húmedos: salinidades de 10 y $36 \mathrm{~g} \mathrm{~kg}^{-1}$ en la época de lluvias y sequía, respectivamente (AgrazHernández, 1999; Riley y Salgado, 1999; Reyes y Tovilla, 2002; Tovilla et al., 2004; Lewis, 2005). La alta salinidad y condiciones xerofitas durante la época seca pueden conducir al enanismo en plantas de mangle (Lin y Sternberg, 1993).

\section{Conclusiones}

En un periodo de dos años, los individuos plantados de Avicennia germinans tuvieron un mejor desempeño que los de Rhizophora mangle, en isletas artificiales producto de dragados en la bahía de Navachiste; este resultado fue debido, principalmente, a que las plantas se establecieron en los mismos niveles topográficos y de inundación donde se encuentran en el manglar original. Finalmente, se encontró que las plantas obtenidas de vivero pueden ser una buena alternativa en la creación de nuevas áreas de manglar, ya que estas presentaron mayor supervivencia y crecimiento en comparación con las extraídas del medio natural.

\section{Agradecimientos}

Se agradece a los revisores anónimos sus comentarios para mejorar el manuscrito. A la Comisión Nacional de Acuacultura y Pesca (CONAPESCA) y al Programa de Mejoramiento del Profesorado de la Secretaría de Educación Pública a través de la Universidad Autónoma de Sinaloa por el apoyo económico, al CONACYT y al Instituto Politécnico Nacional (estudiante PIFI). Además, a la Comisión de Operaciones y Fomento de Actividades Académicas del IPN por la Beca de Exclusividad (SIBE) y por el Estímulo al Desempeño de los Investigadores (EDI).

\section{Literatura citada}

Acosta C.A. y Butler M.J.IV. 1997. Role of mangrove habitat as a nursery for juvenile Spiny lobster, Panulirus argus, in Belize. Marine Freshwater Research 48:721-728.

Agraz-Hernández C.M. 1999. Reforestación experimental de manglares en ecosistemas lagunares estuarinos de la costa noroccidental de México. Tesis Doctoral, Facultad de Ciencias Biológicas, Universidad Autónoma de Nuevo León, Monterrey. 133 pp.

Agraz-Hernández C. 2007. Restauración con manglar: criterios y técnicas hidrológicas de reforestación y forestación. Universidad Autónoma de Campeche. Ciudad del Carmen.

Allen J.A., Krauss K.W. y Hauff R.D. 2003. Factors limiting the intertidal distribution of the mangrove species Xilocarpus granatum. Oecología 135:110-121.

Benitez-Pardo D. 2007. Forestación artificial con manglares en isletas de dragados en una región semiárida de México. Tesis Doctoral, Centro de Interdisciplinario de Ciencias Marinas, Instituto Politécnico Nacional, La Paz, Baja California Sur. 104 pp.

Bosire J.O., Dahdouh-Guebas F., Kairo J.G. y Koedam N. 2003. Colonization of non-planted mangrove species into restored mangrove stands in Gazi Bay, Kenya. Aquatic Botany 76:267-279.

Carrera G.E. y De la Fuente L.G. 2003. Inventario y Clasificación de Humedales en México. Parte I. Ducks Unlimited de México, A.C., México, D. F.

Chapman V.J. 1977. Ecosystems of the World. Vol. I. Wet Coastal Ecosystem. Elsevier Science, Amsterdam.

Clough B.F. 1992. Primary productivity and growth of mangrove forests. En: Robertson A.I. y Alongi D.M. Eds. Coastal and Estuarine Studies. Tropical Mangrove Ecosystems, pp. 225-249, American Geophysical Union, Washington, D.C.

CONAPESCA. Comisión Nacional de Acuacultura y Pesca. 2001. Informe técnico de la reunión de trabajo con pescadores de la Bahía de Navachiste, Guasave, Sinaloa, México. CONAPESCA, Mazatlán.

Cowardin L.M., Carter V., Golet F.C. y LaRoe E.T. 1979. Classification of Wetlands and Deepwater Habitat of the United States. U.S. Department of the Interior, Fish Wildlife Service,Washinton, D.C.

Creary M.M. 2003. Spatial distribution of epibenthic Bryozoans found on the roots of Rhizophora mangle, Kindston, Harbour, Jamaica, W.I. Bulletin of Marine Science 73:477-490.

Delgado P, Hensel P.F, Jiménez J.A. y Day J.W. 2001. The importance of propagule establishment and physical factors in mangrove distributional patterns in a Costa Rican estuary. Aquatic Botany 71:157-178.

Ellison A.M., Mukherjee B.B. y Karim A. 2000. Testing patterns of zonation in mangroves: scale dependence and environmental correlates in the Sundarbans of Bangladesh. Journal of Ecology 88:813-824.

Elster C. 2000. Reason for reforestation success and failure with tree mangrove species in Colombia. Forest Ecology and Management 131:201-214.

FAO. Food and Agriculture Organization of the United Nations. 2007a. The world's mangroves 1980-2005. Forestry Paper 153. Food and Agriculture Organization of the United Nations, Roma.

FAO. Food and Agriculture Organization of the United Nations. 2007b. Los manglares de América del Norte y de América Central 1980-2005. Forest Resources Assessment Programme. 
Working Paper 138. Food and Agriculture Organization of the United Nations, Roma.

Flores-Verdugo F.J. 1989. Algunos aspectos sobre ecología, uso e importancia de los ecosistemas de manglar. En: de la Rosa V.J. y González-Farías F. Eds. Temas de Oceanografía Biológica en México, pp. 21-56, Universidad Autónoma de Baja California. Ensenada.

Flores-Verdugo F.J., Agraz-Hernández C.M. y Benítez-Pardo D. 2006. Creación y restauración de ecosistemas de manglar: principios básicos. En Moreno-Casasola P., Presbarbosa R.E. y Travieso-Bello A.C. Eds. Estrategia para el Manejo Costero Integral: El Enfoque Municipal. 1093-1110. Instituto de Ecología A.C., Comisión Nacional de Áreas Naturales Protegidas, Xalapa.

Flores-Verdugo F., Moreno-Casasola P., Agraz-Hernández C.M., López-Rosas H., Benítez-Pardo D. y Travieso-Bello A.C. 2007. La topografía y el hidroperiodo: dos factores que condicionan la restauración de los humedales costeros. Boletín de la Sociedad Botánica de México 80 (supl.):33-47.

Furukawa K. y Wolanski E. 1996. Sedimentation in mangrove forests. Mangrove and Salt Marshes 1:3-10.

García E. 1980. Modificación del sistema de clasificación climático de Köppen, Instituto de Geografía, Universidad Nacional Autónoma de México, México, D.F.

Hamilton S. 2013. Assessing the role of commercial aquaculture in displacing mangrove forest. Bulletin of Marine Science 89:585601.

Harrison P.J., Snedaker S.C., Ahmed S.I. y Azam F. 1994. Primary producers of the arid climate mangrove ecosystem of the Indus River Delta, Pakistan: An overview. Tropical Ecology 35:155-184.

Hellebust J.A. 1976. Osmoregulation. Annual Review of Plant Physiology 27:485-505.

Jackson M.L. 1964. Análisis Químico de Suelos. Ediciones Omega, Barcelona.

Kieckbusch K.D., Koch M.S., Serafy J.E. y Anderson W.T. 2004. Thophic linkage among primary producers and consumers in fringing mangroves of subtropical lagoons. Bulletin of Marine Science 74:271-285.

Komiyama A., Santiean T., Higo M., Patanaponpaiboon P., Kongsanchai J. y Ogino K. 1996. Microtopography, soil hardness and survival of mangrove (Rhizophora apiculata BL.) seedlings planted in an abandoned tin-minig area. Forest Ecology and Management 81:243-248.

Lewis R.R. 1982. Mangrove forests. En: Lewis R.R. Ed. Creation and Restorations of Coastal Plant Communities, p. 153-171. CRC Press, Boca Raton.

Lewis R.R.III. 2005. Ecological engineering for successful management and restoration of mangrove forests. Ecological Engineering 24:403-418.

Lin G. y Sternberg L.S.L. 1993. Effects of salinity fluctuation on photosynthetic gas exchange and plant growth of the red mangrove (Rhizophora mangle L.) Journal of Experimental Botany 44:9-16.

López-Portillo J. y Ezcurra E. 1989. Response of tree mangroves to salinity in two geoforms. Funtional Ecology 3:355-361.

López-Portillo J., Ewers F.W. y Angeles G. 2005. Sap salinity effects on xylem conductivity in two mangrove species. Plant Cell \& Environment 28:1285-1292.

López-Aguiar L.C. 2006. Distribución espacial y estacional de las principales especies de nitrógeno en aguas de las lagunas costeras de Sinaloa. Tesis de Maestría, Posgrado en Ciencias del Mar y Limnología, Universidad Autónoma de México, Mazatlán. 204 pp.

Lugo A.E. y Cintron G. 1975. The mangrove forest of Puerto Rico and their management. En: Walsh G.E., Snedaker S.C. y Teas H.J. Eds. Proceedings of the International Symposium on Biology and Management Mangroves, p 825-846, Food and Agricultural Sciences, University of Florida, Gainesville.

Mckee K.L., Mendelssohn I.A. y Hester M.W. 1988. Reexamination of pore water sulfide concentrations and redox potentials near the aerial roots of Rhizophora mangle and Avicennia germinans. American Journal of Botany 75:1352-1359.

Medina E., Lugo A.E. y Novelo A. 1995. Contenido mineral del tejido foliar de especies de manglar de la Laguna de Sontecomapan (Veracruz, México) y su relación con la salinidad. Biotropica 27:317-323.

Mendenhall W. y Sincich T. 1997. Probabilidad y Estadística para Ingeniería y Ciencias. Prentice Hall, México D.F.

Monroy-Torres M. 2005. Distribución de tres especies de manglar en relación al hidroperiodo y salinidad intersticial en el estero de Urías, Mazatlán, Sinaloa. Tesis de Licenciatura, Facultad de Ciencias, Universidad Nacional Autónoma de México, México, D.F. 83 pp.

Monzalvo-Santos I.K. 2006. Estimación de la cobertura espacial y análisis de la estructura forestal del manglar en Sinaloa, México, aplicando técnicas de percepción remota. Tesis de Maestría, Instituto de Ciencias del Mar y Limnología, Universidad Nacional Autónoma de México, Mazatlán. 85 pp.

Moreno C.P., Rojas G.J.L., Zarate L.D., Ortiz P.M.A., Lara D.A.L. y Saavedra V.T. 2002. Diagnóstico de los manglares de Veracruz: Distribución, vínculos con los recursos pesqueros y su problemática. Maderas y Bosques Número especial:61-88.

Odum W.E., McIvor C.C. y Smith T.J.III. 1982. The Ecology of Mangroves of South Florida: A Community Profile. U.S. Department of the Interior, Boreau of Land Management, Fish and Wildlife Service, Waslington, D.C.

Paré L. y Fraga J. 1994. La costa de Yucatán: Desarrollo y vulnerabilidad ambiental. Instituto de Investigaciones Sociales, Universidad Nacional Autónoma de México, México, D.F.

Reyes C.M.A. y Tovilla H.C. 2002. Restauración de áreas alteradas de manglar con Rhizophora mangle en la costa de Chiapas. Madera y Bosques. Número especial:103-114.

Riley R.W. y Salgado K.C.P. 1999. Riley encased methodology: principles and processes of mangrove habitat creation and restoration. Mangrove and Salt Marshes 3:207-213.

Ruiz-Fernández A.C., Hillaire-Marcel C., Paéz-Osuna F., Ghaleb B. y Soto-Jímenez M. 2003. Historical trends of metal pollution recorded in the sediments of the Culiacan River Estuary, Northwester Mexico. Applied Geochemistry 18:577-588.

Saenger P. 2002. Mangrove Ecology, Silviculture and Conservation. Kluer Academic Publishers, Dordrecht.

Sáenz R.J.T., Villaseñor R.F.J., Muñoz F.H.J. Rueda S.A. y Prieto R.J.A. 2010. Calidad de planta en viveros forestales de clima templado en Michoacán. Folleto Técnico Número. 17. Instituto Nacional de Investigaciones Forestales, Agrícolas y Pecuarias, Centro de Investigación Regional Pacífico Centro, Campo Experimental Uruapan, Uruapan.

Salisbury F.B. y Ross C. W. 1994. Fisiología Vegetal. Grupo Editorial Iberoamericano, México, D.F. 


\section{Daniel Benitez-Pardo et al.}

SEMARNAP. Secretaría de Medio Ambiente y Recursos Naturales. 2000. Manifestación de impacto ambiental, modalidad particular, para la rehabilitación de la zona estuarina de la bahía de Navachiste, Sinaloa. Secretaría de Medio Ambiente, Recursos Naturales y Pesca, Culiacán.

Toledo G., Rojas A. y Basham Y. 2001. Monitoring of black mangrove restoration with nursery-reared seedlings on an arid coastal lagoon. Hydrobiologia 444:101-109.

Tomlinson P.B. 1986. The Botany of Mangroves. Cambridge University Press, Cambridge.

Tovilla H.C. 1994. Manglares. En: de la Lanza E.G. y Cáceres M.C. Eds. Lagunas Costeras y el Litoral Mexicano, pp. 371423, Universidad Autónoma de Baja California Sur, La Paz.

Tovilla H.C. y Orihuela B.D.E. 2002. Floración, establecimiento de propágulos y supervivencia de Rhizophora mangle L. en el manglar de Barra de Tecoanapa, Guerrero, México. Maderas y Bosques Número especial:89-102.

Tovilla H.C., Román S.A.V., Simuta M.G.M. y Linares M.R.M.
2004. Recuperación del manglar en la barra del río Cahoacán, en la costa de Chiapas. Maderas y Bosques. Número especial 2:77-91.

Turner R.E. 1977. Intertidal vegetation and commercial yields of penaeid shrimp. Trans American Fisheries Society 106:411416.

Yan L. y Guizhu C. 2007. Physiological adaptability of three mangrove species to salt stress. Acta Ecologica Sinica 27:22082214.

Yang S.C, Shih S.S, Hwang G.W., Adams J.B., Lee H.Y. y Chen C.P. 2013. The salinity gradient influences on the inundation tolerance thresholds of mangrove forests. Ecological Engineering 51:59-65.

Zaldívar-Jiménez A., Herrera-Silveira J.A. y Capurro L. 2000. Soil salinity and community structure of two mangrove forests in Yucatan, Southeastern Mexico. Verhandlungen des Internationalen Verein Limnologie 27:1707-1710.

Recibido: 10 de septiembre de 2013

Aceptado: 25 de marzo de 2014 